\title{
FATOU-RIESZ-THEOREMS IN GENERAL SEQUENCE SPACES
}

\section{by GERHARD OTTO MÜLLER and ROLF TRAUTNER}

(Received 1st December 1978)

1.

Consider a formal series $\sum_{n=0}^{\infty} a_{n}$ with partial sums $s_{n}=\sum_{n=0}^{n} a_{k}$ and the corresponding power series $f(z)=\sum_{n=0}^{\infty} a_{n} z^{n}$. Throughout we will assume that $f$ is analytic for $|z|<1$, i.e. that $\limsup _{n \rightarrow \infty}\left|a_{n}\right|^{1 / n} \leqq 1$. A classical theorem of Fatou-Riesz (see $(\mathbf{1}, \mathbf{4})$ ) states that if $\lim _{n \rightarrow 0} a_{n}=0$ and

$$
(F-R): f \text { is analytic for } z=1, f(1)=0
$$

then $\sum_{n=0}^{\infty} a_{n}$ is convergent to 0 .

Jurkat-Peyerimhoff $(2,3)$ obtained the following modification for absolute convergence:

If $\sum_{n=0}^{\infty}\left|a_{n+1}-a_{n}\right|<\infty$ and $(F-R)$ are satisfied then $\sum_{n=0}^{\infty}\left|a_{n}\right|<\infty$.

If we denote by

$$
c_{0}=\left\{a=\left(a_{n}\right)_{0}^{\infty} \mid \lim _{n \rightarrow \infty} a_{n}=0\right\}
$$

the space of null sequences and

$$
b_{v}=\left\{a=\left(a_{n}\right)_{0}^{\infty}\left|\sum_{n=0}^{\infty}\right| a_{n+1}-a_{n} \mid<\infty\right\}
$$

the space of sequences of bounded variation, then the above theorems may be equivalently formulated in the following way:

If $(F-R)$ holds then $\left(a_{n}\right)_{0}^{\infty} \in c_{0}$ implies $\left(s_{n}\right)_{0}^{\infty} \in c_{0}$.

If $(F-R)$ holds then $\left(a_{n}\right)_{0}^{\infty} \in b_{v}$ implies $\left(s_{n}\right)_{0}^{\infty} \in b_{v}$.

This formulation leads to the consideration of a general theorem of the following type:

Given a certain sequence space $V$. If $(F-R)$ holds then $\left(a_{n}\right)_{0}^{\infty} \in V$ implies $\left(s_{n}\right)_{0}^{\infty} \in V$.

The main problem now is to decide for which type of sequence spaces this general 
theorem is valid. Remarkably enough there is a sufficient condition of a purely algebraic nature which applies to a wide class of sequence spaces.

2.

We use the notations $a=\left(a_{n}\right)_{0}^{\infty}, s=\left(s_{n}\right)_{0}^{\infty}$ (where $\left.s_{n}=\sum_{k=0}^{n} a_{k}\right)$ and $e_{k}=\left(\delta_{n k}\right)_{0}^{\infty}$ (having 1 in the $k$-th position and 0 otherwise). We also consider two-sided infinite sequences $g=\left(g_{n}\right)_{-\infty}^{\infty}$. For $\alpha>0$ let

$$
\mathscr{K}_{\alpha}=\left\{g=\left(g_{n}\right)_{-\infty}^{\infty}\left|g_{n}=O\left(|n|^{-\alpha}\right),\right| n \mid \rightarrow \infty\right\}
$$

We consider the convolution product

defined by

$$
b=g * a, \quad\left(b_{n}\right)_{0}^{\infty}=\left(g_{n}\right)_{-\infty}^{\infty} *\left(a_{n}\right)_{0}^{\infty}
$$

$$
b_{n}=\sum_{k=0}^{\infty} g_{n-k} a_{k}, \quad n=0,1,2, \ldots
$$

(the sums being assumed to exist for all $n=0,1,2, \ldots$ ). We will consider a sequence space $V$ which is a linear space over $\boldsymbol{C}$ and in addition satisfies the following axioms:

$A_{0}: \quad e_{0} \in V$

$B$ : for each $a \in V$ there exists $\alpha>0$, such that for every $g=\left(g_{n}\right)_{-\infty}^{\infty} \in \mathscr{K}_{\alpha}$ the condition $g * a=b \in V$ is satisfied.

Axiom $B$ states that $a \in V$ is mapped into $V$ by the convolution product $g * a$, as long as $g_{n}=O\left(|n|^{-\alpha}\right)$.

Here $\alpha=\alpha(a)$ still may depend on the element $a$ considered. However for many spaces $V$ of interest there exists a universal $\alpha_{0}=\alpha_{0}(V)$ for all $a \in V$, i.e. all $g \in \mathscr{K}_{\alpha_{0},}$ act as convolution operators mapping $V$ into itself.

We summarise some simple properties of the space $V$.

(i) For each $a=\left(a_{n}\right)_{0}^{\infty} \in V$ there exists $\beta=\beta(a)>0$ such that $a_{n}=O\left(n^{\beta}\right)$.

This follows from the fact that for $\alpha=\alpha(a)$ the sum $b_{0}=\sum_{k=0}^{\infty}(1+k)^{-\alpha} a_{k}$ exists (take $\beta(a)=\alpha(a))$.

(ii) There exists $\alpha_{1}=\alpha_{1}(V)>0$ such that for

$$
\begin{aligned}
a & =\left(a_{n}\right)_{0}^{\infty} \\
a_{n} & =O\left(n^{-\alpha_{1}}\right) \quad \text { implies } \quad\left(a_{n}\right)_{0}^{\infty} \in V .
\end{aligned}
$$

Take $\alpha_{1}=\alpha\left(e_{0}\right)$ and for $a_{n}=O\left(n^{-\alpha_{1}}\right)$ let

$$
g_{n}=\left\{\begin{array}{cc}
a_{n} & n \geqq 0 \\
0 & n<0
\end{array} ; \text { then } a=g * e_{0} \in V .\right.
$$

Let us denote by $\mathscr{F}$ the set

$$
\left\{g=\left(g_{n}\right)_{-\infty}^{\infty} \mid g_{n} \neq 0 \text { for only finitely many } n \in Z\right\}
$$

Since $g \in \mathscr{F}$ implies $g_{n}=0\left(|n|^{-\alpha}\right)$ for all $\alpha>0$ we get

(iii) $g * a \in V$ for $g \in \mathscr{F}$,

i.e. for $g \in \mathscr{F}$ the convolution operation $g * a$ maps $V$ into itself. 
A particular case is given by

(iv) The shift operators

$$
\begin{aligned}
& \Gamma^{(k)}: a=\left(a_{n}\right)_{0}^{\infty} \rightarrow \Gamma^{(k)} a=\left(0,0, \ldots, 0, a_{0}, a_{1}, \ldots\right) \\
& \text { ( } a_{0} \text { at } k \text {-th position) } \\
& \Gamma^{(-\mathrm{k})}: a=\left(a_{n}\right)_{0}^{\infty} \rightarrow \Gamma^{(-k)} a=\left(a_{k}, a_{k+1}, \ldots\right)
\end{aligned}
$$

( $k \in \boldsymbol{N}$ in both cases) map $V$ into itself.

If we consider the axioms

$$
A_{k}: e_{k} \in V
$$

then we get, from (iv),

(v) If $B$ is assumed, then $A_{0}$ and $A_{k}, k=1,2, \ldots$ are equivalent.

3.

We now are able to state our main result

Theorem 1. Let $V$ be a linear sequence space over the field $\boldsymbol{C}$, satisfying the axioms $A_{0}$ and B. For $a=\left(a_{n}\right)_{0}^{\infty}$ consider the sequence $s=\left(s_{n}\right)_{0}^{\infty}, s_{n}=\sum_{k=0}^{n} a_{k}$, and the power series $f(z)=\sum_{n=0}^{\infty} a_{n} z^{n}$.

If the condition

$$
(F-R): \quad \text { is analytic for } z=1, f(1)=0
$$

is satisfied, then $\left(a_{n}\right)_{0}^{\infty} \in V$ implies $\left(s_{n}\right)_{0}^{\infty} \in V$.

We need the following lemma which is essentially known.

Lemma. Given a function $f$ analytic on the arc

$$
\left\{z=e^{i \phi} \mid \phi_{1} \leqq \phi \leqq \phi_{2}\right\} \quad\left(\phi_{2}<2 \pi+\phi_{1}\right)
$$

having zeros of order $\geqq \gamma \in \mathbf{N}$ at $z_{1}=e^{i \phi_{1}}$ and $z_{2}=e^{i \phi_{2}}$. Then

$$
\int_{\mathscr{S}_{1}}^{\varphi_{2}} f\left(e^{i \phi}\right) e^{i n \phi} d \phi=O\left(|n|^{-\gamma}\right) \text { for }|n| \rightarrow \infty
$$

Proof. There exists $R>1$ such that $f$ is analytic in the closed domain

$$
\left\{z=r e^{i \phi} \mid 1 / R \leqq r \leqq R, \phi_{1} \leqq \phi \leqq \phi_{2}\right\} .
$$

For $n<0$ we write the integral in the form

$$
\begin{aligned}
\frac{1}{i} \int_{e^{i} \phi_{1}}^{e i \phi_{2}} f(z) z^{n-1} d z=\frac{1}{i} & \int_{1}^{R} f\left(r e^{i \phi_{1}}\right) e^{i n \phi_{1}} r^{n-1} d r \\
& +\int_{\phi^{1}}^{\phi_{2}} f\left(R e^{i \phi}\right) R^{n} e^{i n \phi} d \phi+\frac{1}{i} \int_{R}^{1} f\left(r e^{i \phi_{2}}\right) e^{i n \phi_{2}} r^{n-1} d r:=I+I I+I I I .
\end{aligned}
$$


The first integral may be estimated by

$$
|I| \leqq M \int_{1}^{R}(r-1)^{\gamma} r^{-|n|} d r=O\left(|n|^{-\gamma}\right)
$$

the same estimate being valid for the third integral. The second integral is estimated by

$$
|I I| \leqq M R^{-|n|}=O\left(|n|^{-\gamma}\right) \text {. }
$$

For $n>0$ we proceed in a similar way replacing $R$ by $1 / R$.

\section{Proof of Theorem 1. Let}

then

$$
s(z)=\sum_{n=0}^{\infty} s_{n} z^{n}=\frac{1}{1-z} f(z)
$$

$$
s_{n}=\frac{1}{2 \pi i} \oint_{|z|=1 / 2} \frac{s(z)}{z^{n+1}} d z=\frac{1}{2 \pi i} \oint_{|z|=1 / 2} \frac{f(z)}{(1-z)} \cdot \frac{d z}{z^{n+1}} .
$$

Since $f$ is analytic for $z=1$ and $f(1)=0, s$ is analytic for $z=1$, and there exists $0<\phi_{0}<\pi$, such that $s$ is analytic on the arc $\left\{z=e^{i \phi} \mid-\phi_{0} \leqq \phi \leqq \phi_{0}\right\}$.

Now there exists a polynomial $P(z)=\sum_{k=0}^{q} p_{k} z^{k}$ such that

$$
g(z)=\frac{1}{1-z}-P(z)
$$

at $z_{1}=e^{-i \phi_{0}}, z_{2}=e^{+i \phi_{0}}$ has zeros of order $\gamma \geqq \alpha+\alpha_{1}$ where $\alpha=\alpha(a)$ is the exponent from axiom $B$ corresponding to the sequence $a=\left(a_{n}\right)_{0}^{\infty}$ and $\alpha_{1}=\alpha\left(e_{0}\right)$ from property (ii).

We obtain

$$
s_{n}=\frac{1}{2 \pi i} \oint_{|z|=1 / 2} \frac{f(z) P(z) d z}{z^{n+1}}+\frac{1}{2 \pi i} \oint_{|z|=1 / 2} \frac{f(z) g(z) d z}{z^{n+1}}:=s_{n}^{(1)}+s_{n}^{(2)} \text {. }
$$

If we let $p=\left(\ldots 0,0, p_{0}, p_{1}, \ldots, p_{q}, 0, o, \ldots\right)\left(p_{0}\right.$ at 0 -th position $)$ we get $\left(s_{n}^{(1)}\right)_{0}^{\infty}=s^{(1)}=p * a$ which implies $\left(s_{n}^{(1)}\right)_{0}^{\infty} \in V$ by property (iii). In view of property (i) $f$ has distributional boundary values

$$
\left.f(e)^{i \phi}\right)=\sum_{n=0}^{\infty} a_{n} e^{i n \phi}
$$

the sum converging in the distributional sense.

We can therefore write

$$
s_{n}^{(2)}=\frac{1}{2 \pi} \int_{-\phi^{\circ}}^{\phi_{0}} f\left(e^{i \phi}\right) g\left(e^{i \phi}\right) e^{-i n \phi} d \phi+\frac{1}{2 \pi} \int_{\phi_{0}}^{2 \pi-\phi_{0}} f\left(e^{i \phi}\right) g\left(e^{i \phi}\right) e^{-i n \phi} d \phi:=s_{n}^{(3)}+s_{n}^{(4)}
$$

Since $f(z) g(z)$ is analytic on $\left\{z=e^{i \phi} \mid-\phi_{0} \leqq \phi \leqq \phi_{0}\right\}$ having zeros of order $\geqq \gamma$ at $e^{ \pm i \phi_{0}}$ we obtain from the lemma

$$
s_{n}^{(3)}=O\left(n^{-\gamma}\right) \quad n \rightarrow \infty
$$


and $\gamma \geqq \alpha_{1}$ (property (ii)) implies $\left(s_{n}^{(3)}\right)_{0}^{\infty} \in V$. In order to estimate $s_{n}^{(4)}$ let be

$$
h\left(e^{i \phi}\right)=\left\{\begin{array}{cc}
g\left(e^{i \phi}\right), & \phi_{0} \leqq \phi \leqq 2 \pi-\phi_{0} \\
0 & -\phi_{0}<\phi<\phi_{0}
\end{array}\right.
$$

Then $h$ may be represented by a Fourier series

$$
h\left(e^{i \phi}\right)=\sum_{n=-\infty}^{\infty} g_{n} e^{i n \phi} .
$$

Since $g(z)$ is analytic on the $\operatorname{arc}\left\{z=e^{i \phi} \mid \phi_{0} \leqq \phi \leqq 2 \pi-\phi_{0}\right\}$ having zeros of order $\geqq \gamma$ at $e^{ \pm i \phi_{0}}$ we obtain from the lemma

$$
g_{n}=O\left(|n|^{-\gamma}\right) \quad|n| \rightarrow \infty .
$$

Since $f\left(e^{i \phi}\right)$ is analytic for $-\phi_{0} \leqq \phi \leqq \phi_{0}$ and $g\left(e^{i \phi}\right)$ is analytic for $\phi_{0} \leqq \phi \leqq 2 \pi-\phi_{0}$, the product is the well-defined distribution

where

$$
f\left(e^{i \phi}\right) h\left(e^{i \phi}\right)=\sum_{n=-\infty}^{\infty} b_{n} e^{i n \phi}
$$

$$
b_{n}=\frac{1}{2 \pi} \int_{0}^{2 \pi} f\left(e^{i \phi}\right) h\left(e^{i \phi}\right) e^{-i n \phi} d \phi=\sum_{k=0}^{\infty} g_{n-k} a_{k} .
$$

So we obtain $b_{n}=s_{n}^{(4)}$ for $n \geqq 0$ and

$$
\left(s_{n}^{(4)}\right)_{0}^{\infty}=\left(g_{n}\right)_{-\infty}^{\infty} *\left(a_{n}\right)_{0}^{\infty}
$$

which implies $\left(s_{n}^{(4)}\right)_{0}^{\infty} \in V$ from axiom $B$ and $\gamma \geqq \alpha(a)$. From

$$
s_{n}=s_{n}^{(1)}+s_{n}^{(3)}+s_{n}^{(4)}
$$

the statement of the theorem now follows.

4.

We give some examples of sequence spaces for which the Fatou-Riesz-theorem is valid.

Axiom $A_{0}$ is satisfied in nearly all sequence spaces of interest, although there exist trivial counter-examples where it does not hold (take the space spanned by the sequence $(1,1,1, \ldots))$. So the essential point is to show the validity of axiom $B$. In certain cases there exists a universal $\alpha_{0}=\alpha_{0}(V)$ not depending on $a$ such that $B$ is satisfied. This might be checked in the following way. The convolution $b=g * a$ may be formally written as

where

$$
b=S \cdot a
$$

$$
S=\sum_{k=-\infty}^{\infty} g_{k} \Gamma^{(k)}
$$

is an infinite linear combination in the shift operators $\Gamma^{(k)}$.

In order to show that $S$ is a well-defined map from $V$ into itself if $g_{n}=O\left(|n|^{\left.\alpha_{i}\right)}\right.$, one has to show that the operator sum $S=\sum_{k=-\infty}^{\infty} g_{k} \Gamma^{(k)}$ converges in a suitable sense. Usually 
this requires continuity arguments and consequently a topological structure on $V$.

We give a precise statement in the case of a Banach space.

Theorem 2. Let $V$ be a Banach sequence space. Assume that the shift operators $\Gamma^{(k)}$, $k \in \boldsymbol{Z}$, are bounded linear maps from $V$ into itself with norms

$$
\left\|\Gamma^{(k)}\right\|=O\left(|k|^{\beta}\right) \quad \text { as } \quad|k| \rightarrow \infty
$$

for some $\beta>0$.

Then axiom $B$ is satisfied in $V$. If in addition axiom $A$ holds then the Fatou-Riesztheorem is valid in $V$.

Proof. Choose $\alpha_{0}>\beta+1$, then $\left|g_{n}\right|=O\left(|n|^{\left.-\alpha_{0}\right)}\right)$ implies the convergence of $S$ in the operator norm topology.

In the spaces $c_{0}$ and $b_{v}$ we have $\left\|\Gamma^{k}\right\|=1$ and we obtain the known results. We now give some new examples.

(1) Take $V=l_{p}$ the (unweighted) $l_{p}$-space, $1 \leqq p \leqq \infty$, then $\left\|\Gamma^{(k)}\right\|=1$, so Theorem 2 shows the validity of the Fatou-Riesz-theorem. (The case $p=\infty$ was mentioned already by Fatou (1).)

More generally we may take certain weighted $l_{p}$-spaces:

(2) For $1 \leqq p \leqq \infty$ let be

$$
V=l_{w}^{p}=\left\{a=\left(a_{n}\right)_{0}^{\infty},\|a\|_{p, w}=\left\|\left(\frac{a_{n}}{w_{n}}\right)_{0}^{\infty}\right\|_{p}<\infty\right\}
$$

where $\left\|\left(a_{n}\right)_{0}^{\infty}\right\|_{p}$ denotes the usual $l_{p}$-norm, and $w=\left(w_{n}\right)_{0}^{\infty}$ is a fixed positive sequence. Then axiom $B$ is satisfied if the following regularity condition for $w$ holds

$$
\sup _{n=0,1,2, \ldots} \frac{w_{n+k}}{w_{n}}<M \cdot(|k|+1)^{\beta}
$$

for some $M>0, \beta>0$ (where $w_{-n}=w_{0}, n=1,2, \ldots$ ). For we get

$$
\left\|\Gamma^{(-k)} a\right\|_{p, w}=\left\|\left(\frac{w_{n+k}}{w_{n}} \frac{a_{n+k}}{w_{n+k}}\right)\right\|_{p}<\sup _{n=0,1.2 \ldots} \frac{w_{n+k}}{w_{n}}\left\|\frac{a_{n}}{w_{n}}\right\|_{p}<M(|k|+1)^{\beta}\|a\|_{p, w} .
$$

(3) Take the space of $C_{1}$-summable series

$$
C_{1}=\left\{\left(a_{n}\right)_{0}^{\infty}, \lim _{n \rightarrow \infty} \sigma_{n} \text { exists }\right\} \text { where } \sigma_{n}=\frac{1}{n+1} \sum_{k=0}^{n} s_{k}
$$

This is a Banach space with norm $\|a\|=\sup _{n}\left|\sigma_{n}\right|$.

Then $\left\|\Gamma^{(k)}\right\|=0(|k|)$, as seen by direct calculation.

5.

Finally we list some open problems.

1. Conditions for the validity of the Fatou-Riesz-theorem are given by the purely algebraic axioms $A_{0}$ and $B$. Do these axioms imply that the space $V$ can be topologised in a suitable way? 
2. Theorem 1 gives only sufficient conditions for the validity of the Fatou-Riesztheorem, and it remains open whether the theorem could be proved in a much wider class of spaces. Consider

$$
\begin{aligned}
& V_{1}=\left\{a=\left(a_{n}\right)_{0}^{\infty}, \quad \limsup _{n \rightarrow \infty}\left|a_{n}\right|^{1 / n} \leqq 1\right\} \\
& V_{2}=\left\{a=\left(a_{n}\right)_{0}^{\infty}, \quad \limsup _{n \rightarrow \infty} \frac{\log \left|a_{n}\right|}{\log n}<\infty\right\} \\
& V_{3}=\left\{a=\left(a_{n}\right)_{0}^{\infty} \quad f(z)=\sum_{0}^{\infty} a_{n} z^{n} \in H^{p}\right\}
\end{aligned}
$$

(where $H^{p}$ denote the Hardy space of order $p$ ), then $a \in V_{i}$ and $(F-R)$ implies $s \in V_{i}$ as seen directly without using Theorem 1 , in $V_{1}$ and $V_{2}$ it holds even without the $(F-R)$ condition.

3. The classical Fatou-Riesz-theorem has the stronger statement: $\lim _{n \rightarrow \infty} a_{n}=0$ implies the uniform convergence of $s_{n}(z)$ on any closed arc of $|z|=1$ on which $f(z)=\sum_{n=0}^{\infty} a_{n} z^{n}$ is analytic. Does there exist a suitable generalisation in general sequence spaces?

Acknowledgement. After finishing this paper we heard from Professor Jurkat that he had obtained a similar result in connection with the papers published jointly with Professor Peyerimhoff $(2,3)$, but which he did not publish.

\section{REFERENCES}

(1) P. Fatou, Séries trigonométriques et séries de Taylor, Acta Math. 30 (1906), 335-400.

(2) W. Jurkat, A. Peyerimhoff, Der Satz von Fatou-Riesz und der Riemannsche Lokalisationssatz bei absoluter Konvergenz, Arch. Math. 4 (1953), 285-297.

(3) W. JURKat and A. PeYerimhofF, Über einen absoluten Fatou-Rieszschen Satz für Laplaceintegrale, Acad. Serbe Sci. Publ. Inst. Math. 7 (1954), 61-68.

(4) M. RiESZ, Über einen Satz des Herrn Fatou, J. reine angew. Math. 140 (1911), 89-99.

\section{UNIVERSITÄT ULM \\ ABTEILUNG FÜR MATHEMATIK V \\ D-7900 ULM \\ WEST GERMANY}

